\title{
A $q$-ANALOG OF RESTRICTED GROWTH FUNCTIONS, DOBINSKI'S EQUALITY, AND CHARLIER POLYNOMIALS ${ }^{1}$
}

\author{
BY \\ STEPHEN C. MILNE
}

\begin{abstract}
We apply finite operator techniques due to G. C. Rota to a combinatorial identity, which counts a collection of generalized restricted growth functions in two ways, and obtain a q-analog of Charlier polynomials and Dobinski's equality for the number of partitions of an $n$-set. Our methods afford a unified proof of certain identities in the combinatorics of finite dimensional vector spaces over $\mathbf{G F}(q)$.
\end{abstract}

1. Introduction. Let $I_{n}$ be the set of $\{1,2, \ldots, n\}$. A partition of $I_{n}$ is a family of disjoint subsets of $I_{n}$ called "blocks" whose union is $I_{n}$. In [5], Dobinski gave a remarkable formula for the number $B_{n}$ of distinct partitions of $I_{n}$, here and after referred to as $\Pi_{n}$.

$$
B_{n+1}=\frac{1}{e}\left(1^{n}+\frac{2^{n}}{1 !}+\frac{3^{n}}{2 !}+\cdots+\frac{k^{n}}{(k-1) !}+\cdots\right) .
$$

Let $V_{1}$ be the vector space over the reals consisting of all polynomials in the single variable $x$. It turns out that for $p_{1}(u), p_{2}(u) \in V_{1}$,

$$
\left\langle p_{1}(u), p_{2}(u)\right\rangle_{1}=\frac{1}{e} \sum_{k=0}^{\infty} \frac{p_{1}(u) p_{2}(u)}{k !}
$$

is an inner product. If we define $h_{n}(u)$ by

$$
h_{n}(u)=\frac{1}{(n !)^{1 / 2}} \sum_{k=0}^{n}(-1)^{k}\left(\begin{array}{l}
n \\
k
\end{array}\right) u(u-1) \cdots(u-n+k+1),
$$

then

$$
\left\langle h_{n}(u), h_{m}(u)\right\rangle_{1}= \begin{cases}1 & \text { if } n=m, \\ 0 & \text { otherwise. }\end{cases}
$$

These are the classical Charlier polynomials.

Received by the editors May 27, 1977 and, in revised form, September 19, 1977.

AMS (MOS) subject classifications (1970). Primary 05A15, 05C30, 39A10; Secondary 26A86, 50D30.

Key words and phrases. Restricted growth functions, Dobinski's equality, Charlier polynomials, Eulerian derivative, Eulerian generating function, finite operator calculus, finite field, maximal chain, stabilizer groups of a chain, $q$-difference operator, $q$-Stirling numbers of the second kind.

${ }^{1}$ Research partially supported by NSF Grant MCS 74-24249.

() American Mathematical Society 1979 
We now consider a collection of mappings of $I_{n}$ into $I_{n}$ which is in a one-to-one correspondence with $\Pi_{n}$.

DEFINITION 1.1 (RESTRICTED GROWTH FUNCTIONS). A function $f: I_{n} \rightarrow I_{n}$ is said to be of "restricted growth" if and only if

$$
\begin{aligned}
& \text { (a) } f(1)=1, \\
& \text { (b) } f(\nu+1) \leqslant 1+\max _{1<s<\nu} f(s) \text {. }
\end{aligned}
$$

The collection of all restricted growth functions on $I_{n}$ will be referred to as $\operatorname{RG}(n)$.

A one-to-one correspondence of $\Pi_{n}$ onto $\mathrm{RG}(n)$ can be defined as follows. Let $\mathbb{Q}=\left\{A_{1}, A_{2}, \ldots, A_{k}\right\}$ be a $k$-block partition and let $i_{v}$ be the smallest number occurring in $A_{\nu}$. We shall assume that the $A$ 's are labelled in such a manner that $i_{1}, i_{2}, \ldots, i_{k}$ is an increasing sequence. This given, we define $T_{0} \mathscr{Q}$ to be the function $f: I_{n} \rightarrow I_{n}$ which takes the value $\nu$ on the set $A_{\nu}$. It is easy to see that $T_{0} \mathscr{Q}$ is always a restricted growth function and that the map $T_{0}: \Pi_{n} \rightarrow \mathrm{RG}(n)$ is one-to-one and onto. Hence, the number $B_{n}$ in (1.1) also counts the number of maps in $R G(n)$.

Restricted growth functions have been studied in [8], [9] and [11].

The numbers $B_{n}$, Dobinski's equality, and Charlier polynomials are all treated from a finite operator point of view by G. C. Rota in The number of partitions of a set [10]. All three of these objects are directly related to the collection of all mappings of $I_{n}$ into $I_{n}$ by the identity

$$
x^{n}=\sum_{k=0}^{n} S(n, k)(x)_{k}
$$

with $S(n+1, k)=S(n, k-1)+k S(n, k), S(n, k)$ is the number of $k$ block partitions of $I_{n}$, and $(x)_{k}=x(x-1) \cdots(x-k+1)$.

Note that $S(n, k)$ also counts the number of restricted growth functions in $\mathrm{RG}(n)$ with $k$ distinct values.

Definition 1.2. By $\mathcal{L}_{1}(x)$, we mean the collection of all lines in $V_{x}(q)$, the $x$ dimensional vector space over $\operatorname{GF}(q)$. Here $\operatorname{GF}(q)$ is a finite field with $q$ elements, $q$ a power of a prime, and $x$ is a positive integer.

The main stimulus for our work has been to look at the collection of all maps of $I_{n}$ into $\mathscr{L}_{1}(n)$ and find a subcollection which generalizes the restricted growth functions $\mathrm{RG}(n)$ in such a way that an analog of (1.4) holds and for which the finite operator methods of [10] can be applied to yield a "natural" $q$-analog of Dobinski's equality and the Charlier polynomials.

To carry out this program, we make use of the Eulerian derivative, a $q$-analog of the $n$th difference operator, the notion of a "chain associated with a map", and the stabilizer group of a chain of subspaces in a finite dimensional vector space. 
As these notions may be unfamiliar, we present them in a preliminary section. In addition, we state our main results and indicate how they are related to certain identities in the combinatorics of vector spaces over $\operatorname{GF}(q)$.

For our $q$-analog of (1.4), $R G(n)$, Dobinski's equality, and Charlier polynomials, see (1.14), Definition 17, Corollary 1.1 and Theorem 1.3 of the next section.

Preliminaries and statement of results. At this point, it is helpful to recall the definitions of several linear operators on the vector space $V_{1}$ of polynomials in one variable $x$.

Defintion 1.3. For $p(u)$ a polynomial, we let:

$E: \quad p(u) \rightarrow p(u+1)$ be the shift operator,

$D: \quad p(u) \rightarrow p^{\prime}(u)$ be the derivative,

$\Delta \equiv E-I: p(u) \rightarrow p(u+1)-p(u)$ be the difference operator,

$V: \quad f(x) \rightarrow f(1)$ be the linear functional consisting of evaluating a function at $x=1$.

Note that given any operator $L: V_{1} \rightarrow V_{1}$, by $L^{n} f(u)$ we mean $L\left(L^{n-1} f\right)(u)$.

In order to see how to generalize (1.4), we study a $q$-analog of the $n$th difference operator $\Delta^{n}$.

DEFINITION 1.4. Let $q>1$. Then the $q n$th difference operator $\Delta_{q}^{n}$ is defined inductively by means of

$$
\begin{aligned}
\Delta_{q}^{0} f(x) & =I f(x)=f(x), \\
\Delta_{q}^{1} f(x) & =(E-I) f(x)=f(x+1)-f(x), \\
\Delta_{q}^{n+1} f(x) & =\Delta_{q}^{n}\left(E-q^{n} I\right) f(x)=\Delta_{q}^{n} f(x+1)-q^{n} \Delta_{q}^{n} f(x) .
\end{aligned}
$$

Note that $\Delta_{q}^{n} f(x)=\left(E-q^{n-1} I\right)\left(E-q^{n-2} I\right) \cdots(E-I) f(x)$ and $\Delta_{1}^{n} f(x)=(E-I)^{n} f(x)=\Delta^{n} f(x)$.

It follows from the $q$-binomial theorem that

$$
\Delta_{q}^{n} f(x)=\sum_{k=0}^{n}(-1)^{k}\left[\begin{array}{l}
n \\
k
\end{array}\right] q^{\left(\frac{k}{2}\right)} E^{n-k} f(x),
$$

where the following notation is assumed:

$$
\begin{aligned}
{[x] } & =\left(q^{x}-1\right) /(q-1) \\
{[x]_{k} } & =[x][x-1] \cdots[x-k+1], \\
{[n !] } & =[n]_{n}, \quad[0] !=[x]_{0}=1, \quad[x]_{k}=0 \quad \text { if } x<k, x \text { integer, } \\
{\left[\begin{array}{l}
x \\
k
\end{array}\right] } & =[x]_{k} /[k] !, \quad\left[\begin{array}{l}
x \\
0
\end{array}\right]=1 .
\end{aligned}
$$


Note also that

$$
\begin{gathered}
{\left[\begin{array}{l}
n \\
k
\end{array}\right]=\left[\begin{array}{c}
n \\
n-k
\end{array}\right] \quad(0<k<n), \quad\left[\begin{array}{l}
n \\
k
\end{array}\right]=0 \quad(n<k),} \\
{\left[\begin{array}{c}
n+1 \\
k
\end{array}\right]=\left[\begin{array}{c}
n \\
k-1
\end{array}\right]+q^{k}\left[\begin{array}{l}
n \\
k
\end{array}\right] .}
\end{gathered}
$$

In (1.8) and (1.9), $n$ and $k$ are nonnegative integers while $x$ is arbitrary.

We now use the properties of $\Delta_{q}^{n}$ to obtain a $q$-analog of (1.4). Let $U$ be the vector space consisting of all polynomials in the single variable $q^{x}$ with coefficients rational functions in $q$.

The sequence $[x]_{0}=1,[x]_{1}, \ldots,[x]_{k}, \ldots$ is a basis for $U$.

If now $f(x)$ is a polynomial in $q^{x}$ of degree $<n$, since $\left\{[x]_{k}\right\}$ is a basis, we may put

$$
f(x)=\sum_{k=0}^{n} \alpha_{k}[x]_{k}
$$

where $\alpha_{k}$ is a polynomial in $q$, but independent of $x$. To determine the coefficients, we apply $\Delta_{q}^{n}$ to both sides of (1.10). To do this, we need the formula

$$
\Delta_{q}^{n}[x]_{k}=[k]_{n}[x]_{k-n} q^{n(x-k+n)} .
$$

For a proof, note that $\Delta_{q}^{0}[x]_{k}=1 \cdot[x]_{k}$ and do an induction on $n$ while holding $k$ fixed.

It follows that

$$
\alpha_{k}=\Delta_{q}^{k} f(0) /[k] ! .
$$

Note that for $f(x)=x^{n}$ and $q=1$, we would have $\alpha_{k}=\Delta^{k} f(0) / k !=$ $S(n, k)$. In addition, for $q=1,[x]_{k}$ becomes $(x)_{k}$ since $\lim _{q \rightarrow 1^{+}}\left(q^{x}-1\right) /(q$ $-1)=x$. This means that taking $q=1, f(x)=x^{n}$ in (1.10), we would obtain

$$
x^{n}=\sum_{k=0}^{n} S(n, k)(x)_{k}
$$

which is none other than (1.4).

This suggests that to generalize (1.4), we set $f(x)=([x])^{n}$ in (1.10). We then obtain

$$
([x])^{n}=\sum_{k=0}^{n} \frac{\left.\Delta_{q}^{k}([x])^{n}\right|_{x=0}}{[k] !}[x]_{k} .
$$

To this point, our treatment of $\Delta_{q}^{n}$ is due to L. Carlitz in [3]. It is reasonable to generalize $S(n, k)$ for $q>1$ to be simply 
DEFINITION 1.5 ( $q$-STIRLING NUMBERS OF THE SECOND KIND).

$$
S_{q}(n, k)=\frac{\left.\Delta_{q}^{k}([x])^{n}\right|_{x=0}}{[k] !}
$$

Our $q$-analog of (1.4) is then

$$
([x])^{n}=\sum_{k=0}^{n} S_{q}(n, k)[x]_{k} .
$$

From (1.14) follows

$$
\begin{aligned}
([x])^{n+1} & =\sum_{k=0}^{n+1} S_{q}(n+1, k)[x]_{k} \\
& =\sum_{k=0}^{n} S_{q}(n, k)\left([x-k] q^{k}+[k]\right)[x]_{k} \\
& =\sum_{k=0}^{n+1}\left(q^{k-1} S_{q}(n, k-1)+[k] S_{q}(n, k)\right)[x]_{k},
\end{aligned}
$$

which leads to the recursion formula

$$
S_{q}(n+1, k)=q^{k-1} S_{q}(n, k-1)+[k] S_{q}(n, k) .
$$

Formally, a $q$-analog of $B_{n}=\sum_{k=0}^{n} S(n, k)$ is simply

Definition 1.6 ( $q$-ANALOG OF BELL NUMBers $B_{n}$ ).

$$
B_{q, n}=\sum_{k=0}^{n} S_{q}(n, k)
$$

At this point, even through (1.14) is just a formal identity, we have a glimpse of what to do next. As $[x]$ is the number of lines in $V_{x}(q)$, the left-hand side of (1.14) is the number of mappings of $I_{n}$ into $E_{1}(x)$. The right-hand side is indicating how we might sort these maps. $S_{q}(n, k)$ should count the number of generalized restricted growth functions $f: I_{n} \rightarrow \mathfrak{L}_{1}(x)$ for which the span of the lines in the image is a $k$ dimensional subspace of $V_{x}(q)$.

In a moment, the numbers $[x]_{k}$ will play a key role.

The program, outlined after Definition 1.2, that we seek to carry out is directly related to the problem of finding a combinatorial proof of an identity in [3] which is similar to (1.14). The identity is

$$
([x])^{n}=\sum_{k=0}^{n} \tilde{S}_{q}(n, k) q^{\left(\frac{k}{2}\right)}[x]_{k}
$$

with

$$
\tilde{S}_{q}(n+1, k)=\tilde{S}_{q}(n, k-1)+[k] \tilde{S}_{q}(n, k) .
$$

Despite our Definition 1.5 , the numbers $\tilde{S}_{q}(n, k)$ are usually known as $q$-analogues of Stirling numbers of the second kind. They arose in connection with a problem in abelian groups in [4]. 
Ideally, a combinatorial proof of (1.16) would lead to a "natural" collection of objects enumerated by $\tilde{S}_{q}(n, k)$ as well as explain the significance of the factor $q^{\left(\frac{k}{2}\right)}$.

Note the similarity of (1.14) with (1.16) and (1.15) with (1.17). That is $S_{q}(n, k)=q^{\left(\frac{k}{2}\right)} \tilde{S}_{q}(n, k)$. This indicates that the objects counted by the $\tilde{S}_{q}(n, k)$ should be certain equivalence classes of generalized restricted growth functions enumerated by $S_{q}(n, k)$. That is, a combinatorial proof of (1.14) is the first stage in a combinatorial proof of (1.16).

Before we can explain what is involved in our combinatorial proofs of (1.14) and (1.16), we need to make some definitions.

Definition 1.7. Given subspaces $S_{1}, S_{2}, \ldots, S_{l}$ in $V_{x}(q)$, by $\left\langle S_{1}, S_{2}, \ldots, S_{l}\right\rangle$ we mean the span of $S_{1}, S_{2}, \ldots, S_{l}$.

Note that if $y_{1}, y_{2}, \ldots, y_{l}$ are points in $V_{k}(q)$, then $\left\langle y_{1}, \ldots, y_{l}\right\rangle$ is the subspace spanned by $y_{1}, y_{2}, \ldots, y_{l}$.

DefinItION 1.8. Given $f: I_{n} \rightarrow \mathscr{L}_{1}(x), s(f)$ is the dimension of $\langle f(1), f(2), \ldots, f(n)\rangle$.

Definition 1.9. $M(n, x)$ will denote the collection of all mappings $f$ : $I_{n} \rightarrow \varrho_{1}(x)$, with $\varrho_{1}(x)$ the set of all lines in $V_{x}(q)$.

DEFINITION 1.10. $M_{k}(n, x)$ is the collection of mappings

$$
\{f \in M(n, x): s(f)=k\} \text {. }
$$

DEFINITION 1.11. If $(P, \leqslant)$ is a partially ordered set with rank function $r$ and least element 0 , then $\left\{0, x_{1}, x_{2}, \ldots, x_{k}\right\}$ is a complete $k$ chain if and only if

(1) $0<x_{1}<x_{2}<\cdots<x_{k}$,

(2) $r\left(x_{i}\right)=i, 1 \leqslant i \leqslant k$.

Note. What we call a complete $k$ chain is generally known as a maximal chain or "flag" of $(P, \leqslant)$.

We now come to the main construction in our combinatorial proof of (1.14).

Definition 1.12 (Chain associated WITH A MAP). For a mapping $f$ : $I_{n} \rightarrow \mathscr{L}_{1}(x), C(f)$ will denote the complete $k$ chain $\left\{\varnothing \subset E_{1} \subset E_{2} \subset \cdots \subset\right.$ $\left.E_{k}\right\}$, where $k=s(f)$, and for $1 \leqslant l<k$,

$$
E_{l}=\left\langle f(1), f\left(i_{2}\right), \ldots, f\left(i_{l}\right)\right\rangle,
$$

with $1<i_{2}<i_{3}<\cdots<i_{k} \leqslant n$, chosen so that $\operatorname{dim} E_{l}=l, f(i) \subseteq E_{l}$ for $i<i_{l+1}$, and $f\left(i_{l+1}\right) \& E_{l}$.

We will refer to $C(f)$ as the chain associated with the mapping $f$, and say that $f$ is associated with the chain $C(f)$. Note that given $f$, the chain $C(f)$ is uniquely determined.

The main idea in the proof of (1.14) is to split up $M_{k}(n, x)$ into equivalence classes by the following equivalence relation. 
Definition 1.13. Given $f, g \in M_{k}(n, x)$, we say that $f \sim_{1} g$ if and only if $C(f)=C(g)$.

The equivalence classes of $\sim_{1}$ in $M_{k}(n, x)$ are

Definition 1.14. Given $E=\left\{E_{1} \subset E_{2} \subset \cdots \subset E_{k}\right\}$, a complete $k$ chain in $V_{x}(q), M_{k}(n, x, E)$, is the collection of maps $\left\{f \in M_{k}(n, x): C(f)=E\right\}$.

$M_{k}(n, x, E)$ will be known as the collection of maps associated with the chain $E$.

Our proof of (1.14) essentially depends on the fact that the number of complete $k$ chains in $V_{x}(q)$ is $[x]_{k}$ and that the number of maps $f: I_{n} \rightarrow \mathscr{L}_{1}(x)$ associated with any fixed $k$ chain is $S_{q}(n, k)$.

The additional concept that we need to put together a combinatorial proof of (1.16) is provided by

Definition 1.15 (SUBSTABILIZER GROUP OF A MAP). Given $f: I_{n} \rightarrow \mathscr{L}_{1}(x)$, $U_{C(f)}$ will be the group of all one-to-one, linear transformations, with eigenvalues 1 that fix $C(f)$. That is, all such $T:\langle f(1), \ldots, f(n)\rangle \mapsto$ $\langle f(1), \ldots, f(n)\rangle$, so that if $C(f)=\left\{\varnothing \subset E_{1} \subset E_{2} \subset \cdots \subset E_{k}\right\}$, then $T\left(E_{l}\right)$ $=E_{l}, 1 \leqslant l \leqslant k$.

Recalling that $S_{q}(n, k)=\tilde{S}_{q}(n, k) q^{\left(\frac{k}{2}\right)}$, the essential idea in our proof of (1.16) is to split up each $\sim_{1}$ equivalence class of $M_{k}(n, x)$ into equivalence classes with the help of Definition 1.15. That is, for $f \in M_{k}(n, x)$, we split up $M_{k}(n, x, C(f))$ into equivalence classes by means of

Definition 1.16. Given $g, h \in M_{k}(n, x, C(f))$, we say that $g \sim_{2} h$ if and only if $g=T \circ h$, where $T \in U_{C(f)}$.

The main additional fact responsible for (1.16) is that given any $f \in$ $M_{k}(n, x)$, each $\sim_{2}$ equivalence class of $M_{k}(n, x, C(f))$ has $q^{\left(\frac{k}{2}\right)}$ mappings in it and there are $\tilde{S}_{q}(n, k)$ such equivalence classes. The significance of $q^{\left(\frac{k}{2}\right)}$ it that it is the order of the group $U_{C(f)}$.

It is now not difficult to find a subcollection of $M(n, n)$ which generalizes the collection $\mathrm{RG}(n)$ of restricted growth functions of $I_{n}$ into $I_{n}$. Just choose a fixed $n$ complete chain of the vector space $V_{n}(q)$, say $\left\{E_{1} \subset E_{2} \subset \cdots \subset\right.$ $\left.E_{n}\right\}$ and just consider the maps $f: I_{n} \rightarrow \mathscr{L}_{1}(n)$ whose chain is of the form $\left\{E_{1} \subset E_{2} \subset \cdots \subset E_{k}\right\}$. We formalize this by

DEFINITION 1.17 (RESTRICTED GROWTH FUNCTIONS OF $M(n, n)$ ). Given a fixed $n$ complete chain $\left\{E_{1} \subset E_{2} \subset \cdots \subset E_{n}\right\}=E$ of $V_{n}(q)$, by $\mathrm{RG}(n, q, E)$ we mean the collection

$$
\left\{f \in M(n, n): C(f)=\left\{E_{1} \subset E_{2} \subset \cdots \subset E_{k}\right\}, s(f)=k\right\} .
$$

We call $\operatorname{RG}(n, q, E)$ the restricted growth functions of $M(n, n)$ relative to the chain $E$.

By Definition 1.17 and our remark about the proof of (1.14) following Definition 1.14, $S_{q}(n, k)$ counts the number of $f \in \operatorname{RG}(n, q, E)$ with $s(f)=$ $k$. 
Applying our definitions to the subset case, it is not difficult to see that $\mathrm{RG}(n)$ is simply those maps $f: I_{n} \rightarrow I_{n}$ such that $C(f)=\left\{S_{1} \subset S_{2} \subset \cdots \subset\right.$ $\left.S_{k}\right\}$, with $S_{i}=\{1,2, \ldots, i\}$ and $S(f)=k=$ the number of distinct values of $f$.

So, indeed, $\operatorname{RG}(n, q, E)$ is a generalization of $\operatorname{RG}(n)$. In a strict sense, we are generalizing $\operatorname{RG}(n)$ but not $\Pi_{n}$.

Before indicating how the finite operator methods of [10] can be applied to (1.14) to give us our $q$-analogs of Dobinski's equality and Charlier polynomials, we make several remarks about our constructions behind the proofs of (1.14) and (1.16).

Our proof of (1.14) has a direct counterpart that gives a new way of establishing (1.4). Let $V_{x}(1)$ be the lattice of subsets of an $x$ element set. If $y_{1}, y_{2}, \ldots, y_{l}$ are elements of $I_{x}$, then by $\left\langle y_{1}, y_{2}, \ldots, y_{l}\right\rangle$ we mean the set $\left\{y_{1}, y_{2}, \ldots, y_{l}\right\} \subseteq I_{x}$. Interpreting our definitions with this change, it turns out that the number of complete $k$ chains in $V_{x}(1)$ is simply $(x)_{k}=x(x-$ 1) $\cdots(x-k+1)$ and the number of maps $f: I_{n} \rightarrow I_{x}$ associated with any fixed $k$ chain in $V_{x}(1)$ is $S(n, k)$. That is, there are $(x)_{k} \sim_{1}$ equivalence classes with $S(n, k)$ elements in each. Since every map has a unique $k$ chain, we obtain (1.4).

The only use that has been made of the domain $I_{n}$ is to regard each mapping $f: I_{n} \rightarrow I_{x}$ as an ordered set of $n$ points in $I_{x}$. Our equivalence relation $\sim_{1}$ is formulated by Definition 1.13 in terms of the range $I_{x}$.

The usual combinatorial proof of (1.4) is the "dual" of the one we have given here. Rather than splitting up our collection of mappings by an equivalence relation $\sim_{1}$ essentially constructed in terms of $\operatorname{RG}(n)$ and $I_{x}$, it instead uses an equivalence relation $\sim_{\pi}$ constructed in terms of $\Pi_{n}$ and $I_{n}$.

To every function $f: I_{n} \rightarrow I_{x}$ there is associated a partition $\Pi$ of the set $I_{n}$, called the kernel of $f$, defined as follows. Two elements $a$ and $b$ of $I_{n}$ belong to the same block of $\Pi$ if and only if $f(a)=f(b)$. We say $f \sim_{\pi} g$ if and only if $f$ and $g$ have the same kernel. Equivalence classes are all maps with the same kernel. (1.4) now follows directly from the fact that there are $S(n, k)$ different $\sim_{\pi}$ equivalence classes corresponding to $k$-block partitions of $I_{n}$ and that the number of maps in any of these $\sim_{\pi}$ equivalence classes is the same as the number of one-to-one maps of $I_{k}$ into $I_{x}$. This number is simply $(x)_{k}=x(x-1) \cdots(x-k+1)$. Note that there are $S(n, k) \sim_{\pi}$ equivalence classes with $(x)_{k}$ elements in each, and that $\sim_{\pi}$ is defined in terms of $\Pi_{n}$ and $I_{n}$.

This is just the reverse of what our combinatorial proof specializes to. Our concept of a chain associated with a map $f: I_{n} \rightarrow I_{x}$ plays the role of the concept of a one-to-one map. This suggests that other combinatorial proofs can be reformulated in their "dual" form and that perhaps several identities 
with no known combinatorial interpretation can be given one by our methods.

As an example, we reformulate the usual combinatorial proof of a $q$ binomial theorem which is the starting point for [6], and show how the two proofs are related.

Consider the collection of all mappings of $I_{n}$ into the points of $V_{x}(q)$. (We are now looking at mappings into the points of an affine space rather than the points of a projective space.) Let $\mathscr{E}_{0}(x)$ denote the set of points in $V_{x}(q)$. Definitions 1.7 through 1.17 are unchanged as we go from the projective to affine space with one exception. In Definition 1.15, which defines the substabilizer group of a map, the condition that the eigenvalues be 1 is dropped. This gives,

Definition 1.18 (STABILIzer Group of A MAP). Given $f: I_{n} \rightarrow \mathscr{L}_{0}(x), S_{C(f)}$ will be the group of all one-to-one, linear transformations that fix $C(f)$.

Just as in our proof of (1.16), we have the fact that given any $f \in M_{k}(n, x)$, each $\sim_{2}$ equivalence class of $M_{k}(n, x, C(f))$ has $(q-1)^{k} q^{\left(\frac{k}{2}\right)}$ mappings in it and there are $\left[\begin{array}{l}n \\ k\end{array}\right]$ such equivalence classes. The significance of $(q-1)^{k} q^{\left(\frac{k}{2}\right)}$ is that it is the order of the group $S_{C(f)}$.

The number of $k$ complete chains of $V_{x}(q)$ is still $[x]_{k}$ and the number of points is $q^{x}$. Therefore, we obtain,

$$
\left(q^{x}\right)^{n}=\sum_{k=0}^{n}\left[\begin{array}{l}
n \\
k
\end{array}\right](q-1)^{k} q^{\left(\frac{k}{2}\right)}[x]_{k} .
$$

This is equivalent to the identity,

$$
\left(q^{x}\right)^{n}=\sum_{k=0}^{n}\left[\begin{array}{l}
n \\
k
\end{array}\right]\left(q^{x}-1\right)\left(q^{x}-q\right) \cdots\left(q^{x}-q^{n-k-1}\right) .
$$

(1.19) is the starting point for [6].

Before we sketch the proof given in [6] of (1.19), we need one definition.

DEFINITION 1.19. Fix an ordered basis $\left\{y_{1}, y_{2}, \ldots, y_{n}\right\}$ of $V_{n}(q)$. Given any mapping $f: I_{n} \rightarrow \complement_{0}(x)$, we define the map $\Gamma f: V_{n}(q) \rightarrow V_{x}(q)$ by setting

$$
\Gamma f\left(y_{i}\right)=f(i) \text {. }
$$

We say that $\Gamma f$ is the "linearized" version of $f$.

The gist of the proof in [6] of (1.19) is that there are $\left(q^{x}-1\right)\left(q^{x}-\right.$ $q) \cdots\left(q^{x}-q^{n-k-1}\right)$ mappings of $V_{n}(q)$ into $V_{x}(q)$ with any given $k$ dimensional null space. Since each such map has a unique null space and the number of $k$ dimensional subspaces of $V_{n}(q)$ is $\left[\begin{array}{c}n \\ k\end{array}\right]$, we immediately have (1.19).

It turns out that our notion of $S_{C(f)}$ is related to the null space of $\Gamma g$, $g \in M_{k}(n, x, C(f))$, by

Proposition 1.1. Given $g, h \in M_{k}(n, x, C(f))$, there exists a $T \in S_{C(f)}$ 
such that $h=T \circ g$ if and only if the null spaces of $\Gamma h$ and $\Gamma g$ are the same.

In our proof of (1.18), the notions of a chain associated with a map and stabilizer group of a map replace the concept of null space used in (1.19). Here our proof is more complex than the one given in [6. However, we gain the advantage of having a common method of proof for identities (1.4), (1.14), (1.16) and (1.18).

We finally indicate how the finite operator methods of [10] can be applied to (1.14).

Recall that $\left\{[x]_{k}\right\}$ is a basis for the vector space $U$ consisting of all polynomials in $q^{x}$ whose coefficients are rational functions in $q$. Applying the main idea of [10] to $U$, we note that there is a unique linear functional $L_{q}$ on $U$ such that

$$
L_{q}(1)=1, \quad L_{q}\left([x]_{k}\right)=1, \quad k=1,2,3, \ldots
$$

Applying $L_{q}$ to both sides of (1.14), we obtain

$$
L_{q}\left([x]^{n}\right)=\sum_{k=0}^{n} S_{q}(n, k)=B_{q, n} .
$$

As in [10], we generalize Dobinski's equality and the Charlier polynomials by exploiting (1.21) and other properties of $L_{q}$. The proofs of our results will be similar except for the following crucial differences.

We use the $n$th $q$ difference operator $\Delta_{q}^{n}$ instead of the $n$th difference operator $\Delta^{n}$.

The Eulerian derivative $D_{q}$ replaces the usual derivative operator $D$.

DEFINITION 1.20. Let $p(x)$ be a polynomial. Then the Eulerian derivative operator $D_{q}$ is defined by means of

$$
D_{q} p(x)=\frac{p(q x)-p(x)}{(q-1) x} .
$$

Note that $\lim _{q \rightarrow 1^{+}} D_{q} p(x)=p^{\prime}(x)$.

There are two $q$-analogs of the exponential function $e^{x}$ in common use. They are

$$
e(x)=\sum_{k=0}^{\infty} \frac{x^{k}}{[k] !}
$$

and

$$
E(x)=\sum_{k=0}^{\infty} \frac{q^{k(k-1) / 2} x^{k}}{[k] !} .
$$

Note that $(e(x))^{-1}=E(-x)$.

Our $q$-analog of Dobinski's equality will be a special case of: 
THEOREM 1.1. For $p(u)$ any polynomial in $q^{u}$,

$$
L_{q}(p(u))=\frac{1}{e(1)} \sum_{k=0}^{\infty} \frac{p(k)}{[k] !} .
$$

By setting $p(u)=[u]^{n+1}$ in Theorem 1.1 and noting (1.21), we obtain

COROLLARY 1.1 ( $q$-DOBINSKI EQUALITY).

$$
B_{q, n+1}=\frac{1}{e(1)}\left([1]^{n}+\frac{[2]^{n}}{[1] !}+\frac{[3]^{n}}{[2] !}+\ldots\right)
$$

Note the similarity with the classical Dobinski's formula in (1.1).

Define a step function $P_{q}(x)$ by means of

$$
P_{q}(x)=\sum_{\substack{k<x \\ k=0,1,2, \ldots}} \frac{1}{e(1)} \frac{1}{[k] !} .
$$

There is a jump of $(1 / e(1))(1 /[k] !)$ at $x=k$.

By the definition of Riemann-Stieltjes integration and Dobinski's equality, we obtain

$$
B_{q, n}=\int_{-\infty}^{+\infty}[x]^{n} d P_{q}(x) .
$$

This means that our $q$-Bell numbers $B_{q, n}$ are the $n$th moments of a $q$-Poisson distribution, $P_{q}(x)$.

Indeed, for $q=1$, we would have

$$
B_{n}=\int_{-\infty}^{+\infty} x^{n} d P(x)
$$

with

$$
P(x)=\sum_{\substack{k<x \\ k=0,1,2, \ldots}} \frac{1}{e} \frac{1}{k !},
$$

which is just a Poisson distribution with $\lambda=1$.

It turns out that $\int_{-\infty}^{+\infty}[x]_{n} d P_{q}(x)=1=L_{q}\left([x]_{n}\right)$. Starting from this identity and (1.14), it is not difficult to derive our $q$-analog of Dobinski's equaltiy directly.

Note that Theorem 1.1 shows that $L_{q}$ is positive definite on the half-line $[0, \infty)$. This means that we may define an inner product for the vector space $U$ in terms of the linear functional $L_{q}$ by

$$
\left\langle P_{1}(u), P_{2}(u)\right\rangle_{q}=L_{q}\left(P_{1}(u) P_{2}(u)\right) \text {. }
$$

Our $q$-analog of the classical Charlier polynomials will be a consequence of the fundamental 
THEOREM 1.2. For $p(u)$ any polynomial in $q^{u}$ and $g$ any polynomial in $x$, we have the identity,

$$
L_{q}(g(E) p(u))=L_{q}\left(p(u) V g\left(D_{q}\right) x^{u}\right) .
$$

By setting $g(x)=\left(x-q^{n-1}\right)\left(x-q^{n-2}\right) \cdots(x-1)$ in Theorem 1.2 and noting that $g(E)$ is the $q n$th difference operator $\Delta_{q}^{n}$, one can derive

Theorem 1.3 ( $q$-ChARLIER POlynomials). Set

$$
h_{q, n}(u)=\frac{1}{f_{q}(n)^{1 / 2}} \sum_{k=0}^{n}(-1)^{k}\left[\begin{array}{l}
n \\
k
\end{array}\right] q^{\left(\frac{k}{2}\right)}[u]_{n-k},
$$

where

$$
f_{q}(n)=[n] ! \sum_{k=0}^{n}\left(\begin{array}{l}
n \\
k
\end{array}\right)(q-1)^{k} B_{q, k}
$$

Then

$$
\left\langle h_{q, n}(u), h_{q, m}(u)\right\rangle_{q}= \begin{cases}1 & \text { if } n=m, \\ 0 & \text { otherwise. }\end{cases}
$$

Note that $\lim _{q \rightarrow 1^{+}} h_{q, n}(u)=h_{n}(u)$, the Charlier polynomial given (1.2).

In the combinatorial results section, we present the details of the combinatorial proofs of identities (1.14), (1.16) and Proposition 1.1.

The following section on finite operator methods contains proofs of Theorems 1.1, 1.2 and 1.3.

Finally, in the last section on generating functions, we deal with $q$-analogs of the exponential generating function

$$
\sum_{n=0}^{\infty} \frac{B_{n}}{n !} t^{n}=e^{\left(e^{t}-1\right)}
$$

To this end we state one particularly useful estimate for $B_{q, n}$ that may be derived from a maximal term analysis of Dobinski's equality.

THEOREM 1.4. For $q>1$ there exists a constant $k_{q}$ depending only on $q$, so that for all $n$ large enough the following inequalities hold:

$$
\frac{\left[n-k_{q}\right]^{n}}{\left[n-k_{q}\right] !} \leqslant e(1) B_{q, n} \leqslant(2 n+2) \frac{\left[n-k_{q}\right]^{n}}{\left[n-k_{q}\right] !} .
$$

It turns out that for $q \geqslant 2, k_{q}=0$, and $k_{q} \rightarrow \infty$ as $q \rightarrow 1^{+}$.

2. Combinatorial results. As noted after Definition 1.14 in the introduction, in order to prove (1.14), we first give a combinatorial proof of

LEMMA 2.1. Given any $k$ chain $E=\left\{\varnothing \subset E_{1} \subset \cdots \subset E_{k}\right\}$ of $V_{x}(q)$, 
$\left\|M_{k}(n, x, E)\right\|$ depends only on $n$ and $k$. Indeed, $\left\|M_{k}(n, x, E)\right\|=S_{q}(n, k){ }^{2}$

Proof. We first show that $\left\|M_{k}(n, x, E)\right\|$ depends only on $n$ and $k$. To this end, let $E=\left\{E_{1} \subset E_{2} \subset \cdots \subset E_{k}\right\}$ and $F=\left\{F_{1} \subset F_{2} \subset \cdots \subset F_{k}\right\}$ be two different complete $k$ chains of $V_{x}(q)$. Suppose that $\left\{y_{1}, \ldots, y_{k}\right\}$ and $\left\{z_{1}, \ldots, z_{k}\right\}$ are ordered bases of $E$ and $F$, respectively, such that $E_{i}=$ $\left\langle y_{1}, y_{2}, \ldots, y_{i}\right\rangle$ and $F_{i}=\left\langle z_{1}, \ldots, z_{i}\right\rangle$.

We define $\varphi: E_{k} \mapsto F_{k}$ by setting,

Definition 2.1. $\varphi\left(y_{i}\right)=z_{i}, 1 \leqslant i \leqslant k$.

It is clear that $\varphi$ is a linear, one-to-one correspondence of $E_{k}$ onto $F_{k}$ such that $E_{i+1}-E_{i}$ is mapped one-to-one onto $F_{i+1}-F_{i}$.

Definition 2.2. Define $\tilde{\varphi}: M_{k}(n, x, E) \mapsto M_{k}(n, x, F)$ by setting, for $f \in$ $M_{k}(n, x, E)$,

$$
\tilde{\varphi}(f)=\varphi \circ f .
$$

By Definition 1.12 of the Introduction and the fact that $\varphi\left(\left\langle\sum_{i=1}^{k} a_{i} y_{i}\right\rangle\right)=$ $\left\langle\sum_{i=1}^{k} a_{i} z_{i}\right\rangle$, it follows that $\tilde{\varphi}$ is well defined. By construction, $\tilde{\varphi}$ is one-to-one. For if not, then there exists an $h \in M_{k}(n, x, F)$ and $f_{1} \neq f_{2}$ in $M_{k}(n, x, E)$ such that $\varphi \circ f_{1}=\varphi \circ f_{2}=h$. But this means there exist $w_{1}, w_{2} \in E_{k}$ such that $w_{1} \neq w_{2}$ and $\varphi\left(w_{1}\right)=\varphi\left(w_{2}\right)$. Thus, $\varphi$ would not be one-to-one, a contradiction. $\tilde{\varphi}$ is onto since $\varphi$ gives a bijection between the set of lines of $E_{k}$ and those of $F_{k}$.

Therefore $\tilde{\varphi}$ is a one-to-one correspondence of $M_{k}(n, x, E)$ onto $M_{k}(n, x, F)$ which shows that $\left\|M_{k}(n, x, E)\right\|$ depends only on $n$ and $k$.

Note that we did not make use of the linearity of $\varphi$. This property will be used in the proof of Proposition 1.1.

For convenience, let $\left\|M_{k}(n, x, E)\right\|=F(n, k)$. We calculate $F(n, k)$ by showing that it satisfies the difference equation given in (1.15) for $S_{q}(n, k)$. Since the initial conditions for $F(n, k)$ and $S_{q}(n, k)$ are the same and (1.15) has a unique solution, this will show that $\left\|M_{k}(n, x, E)\right\|=S_{q}(n, k)$. We give a direct combinatorial argument.

It is sufficient to work with a fixed complete $n+1$ chain $E=\left\{E_{1} \subset E_{2}\right.$ $\left.\subset \cdots \subset E_{n+1}\right\}$ of $V_{n+1}(q)$. By $F_{k}$ we mean the chain $\left\{E_{1} \subset E_{2} \subset \cdots \subset\right.$ $\left.E_{k}\right\}$. For $f \in M_{k}\left(n+1, n+1, F_{k}\right), \tilde{f}$ will denote the restriction of $f$ to $I_{n}$.

There are only two cases to consider.

Case 1. $S(\tilde{f})=k-1$ and $C(\tilde{f})=F_{k-1}$. That is, $\tilde{f} \in M_{k-1}(n, n+$ $\left.1, F_{k-1}\right)$. Since $f \in M_{k}\left(n+1, n+1, F_{k}\right)$, then $f(n+1)$ must be a line in $E_{k}-E_{k-1}$. As $E_{i}$ has $[i]$ lines, there are only $q^{k-1}$ choices for $f(n+1)$.

Case 2. $S(\tilde{f})=k$ and $C(\tilde{f})=F_{k}$. That is, $\tilde{f} \in M_{k}\left(n, n+1, F_{k}\right)$. Then $f(n+1)$ can be any line in $E_{k}$. So there are $[k]$ choices for $f(n+1)$.

\footnotetext{
${ }^{2}$ For a finite set $X$, by $\|X\|$ we mean the cardinality of $X$.
} 
Putting both cases together, we have $F(n+1, k)=q^{k-1} F(n, k-1)+$ $[k] F(n, k)$.

This finishes the proof of the lemma.

Though well known, we now compute the number of complete $k$ chains in $V_{x}(q)$.

First, define an equivalence relation on the collection of all the ordered bases of all the $k$ dimensional subspaces of $V_{x}(q)$ by means of the following relation.

The ordered bases $y=\left(y_{1}, y_{2}, \ldots, y_{k}\right)$ and $z=\left\{z_{1}, z_{2}, \ldots, z_{k}\right\}$ are equivalent if and only if $C(y)=C(z)$, where $C(y)$ is the complete $k$ chain

$$
\left\{\left\langle y_{1}\right\rangle \subset\left\langle y_{1}, y_{2}\right\rangle \subset \cdots \subset\left\langle y_{1}, y_{2}, \ldots, y_{k}\right\rangle\right\} \text {. }
$$

For any $k$ chain $E=\left\{E_{1} \subset E_{2} \subset \cdots \subset E_{k}\right\}$, there are $q^{k-1}$. $q^{k-2} \cdots q^{1}=q^{\left(\frac{k}{2}\right)}$ ways to choose an ordered basis $y$ of $E_{k}$ so that $C(y)$ is the chain $E$ which we started with.

On the other hand, there are $[x]_{k} q^{\left(\frac{k}{2}\right)}$ ordered bases with $k$ elements chosen from $V_{x}(q)$.

Therefore, the number of equivalence classes, which is the same as the number of $k$ complete chains of $V_{x}(q)$, is

$$
[x]_{k} q^{\left(\frac{k}{2}\right)} / q^{\left(\frac{k}{2}\right)}=[x]_{k} .
$$

We now put together a proof of (1.14).

By Definition 1.13 and (1.14) of the Introduction, Lemma 2.1 and our calculation of the number of $k$ chains in $V_{x}(q)$, we have the fact that $M_{k}(n, x)$ splits up into $[x]_{k} \sim_{1}$ disjoint equivalence classes with $S_{q}(n, k)$ mappings in each class. Therefore,

$$
\left\|M_{k}(n, x)\right\|=S_{q}(n, k)[x]_{k} .
$$

We immediately have

$$
[x]^{n}=\|M(n, x)\|=\sum_{k=0}^{n}\left\|M_{k}(n, x)\right\|=\sum_{k=0}^{n} S_{q}(n, k)[x]_{k} \text {, which is (1.14). }
$$

Recalling Definition 1.16, our first step in the proof of (1.16) will be to show that given any $f \in M_{k}(n, x)$, each $\sim_{2}$ equivalence class of $M_{k}(n, x, C(f))$ has $q^{\left(\frac{k}{2}\right)}$ mappings in it. By Lemma 2.1, $\left\|M_{k}(n, x, C(f))\right\|$ equals $S_{q}(n, k)$. Therefore, the number of $\sim_{2}$ equivalence classes of $M_{k}(n, x, C(f))$ depends only on $n$ and $k$. Then, by the same methods as in the second half of Lemma 2.1, we show that this number is $\tilde{S}_{q}(n, k)$. But, this means that for any complete $k$ chain $E$ of $V_{x}(q)$, we have $\left\|M_{k}(n, x, E)\right\|=$ $\tilde{S}_{q}(n, k) q^{\left(\frac{k}{2}\right)}$. Since the $M_{k}(n, x, E)$ are the $\sim_{1}$ equivalence classes of $M_{k}(n, x)$,

$$
\sum_{E}\left\|M_{k}(n, x, E)\right\|=\left\|M_{k}(n, x)\right\|
$$


where the sum is over all complete $k$ chains of $V_{x}(q)$. Note that there are $[x]_{k}$ such chains. Putting all these facts together, we obtain

$$
\begin{aligned}
{[x]^{n} } & =\sum_{k=0}^{n}\left\|M_{k}(n, x)\right\| \\
& =\sum_{k=0}^{n} \sum_{E}\left\|M_{k}(n, x, E)\right\|=\sum_{k=0}^{n} \sum_{E} \tilde{S}_{q}(n, k) q^{\left(\frac{k}{2}\right)} \\
& =\sum_{k=0}^{n} \tilde{S}_{q}(n, k) q^{\left(\frac{k}{2}\right)}[x]_{k},
\end{aligned}
$$

which is (1.16).

Now we show that each $\sim_{2}$ equivalence class has $q^{\left(\frac{k}{2}\right)}$ elements.

Recall Definitions 1.15 and 1.16 from the Introduction. That is, two maps $g, h \in M_{k}(n, x, C(f))$ are equivalent if and only if $g=T \circ h$, where $T$ is some linear, one-to-one transformation, with eigenvalues 1 , which fixes the subspaces in the chain $C(f)$. We indicate this by $g \sim_{2} h$.

We may express the $k$ chain $C(f)$ as, $\left\{E_{1} \subset E_{2} \subset \cdots \subset E_{k}\right\}$, where $E_{i}=\left\langle y_{1}, \ldots, y_{i}\right\rangle$, with $y=\left\{y_{1}, y_{2}, \ldots, y_{k}\right\}$ an ordered basis of $E_{k}$.

What do the transformations $T$ look like? Represent $T$ by the matrix $\left(a_{i j}\right)$, $1 \leqslant i, j \leqslant k$, where $T y_{i}=\sum_{s=1}^{k} a_{s i} y_{s}$.

It is not hard to see that since $T\left(E_{i}\right)=E_{i}$, for $1 \leqslant i \leqslant k$, we have $a_{i j}=0$ if $i>j$. That is, $T$ has zero entries below the diagonal. Since $T$ is one-to-one, we must have nonzero entries on the diagonal. Finally, since the eigenvalues are $1, T$ has just l's on the diagonal.

There are $q^{\left(\frac{k}{2}\right)}$ possible such transformations $T$ as each of the $\left(\begin{array}{l}k \\ 2\end{array}\right)$ entries above the diagonal can be any one of the $q$ elements of the field $\operatorname{GF}(q)$.

This collection of matrices forms a group under matrix multiplication. This implies that $\sim_{2}$ as defined above is an equivalence relation on the set $M_{k}(n, x, C(f))$.

Each equivalence class has $q^{\left(\frac{k}{2}\right)}$ elements. This follows from the fact that given any $g \in M_{k}(n, x, C(f))$ and $T_{1}, T_{2}$ in the group $U_{C(f)}, T_{1} \circ g=T_{2} \circ g$ implies that $T_{1}=T_{2}$. This is because among the images of $g$ there is a basis of $E_{k}$, so that $T_{1}$ and $T_{2}$ agree on a basis.

As in the last half of Lemma 2.1, we now show that the number of $\sim_{2}$ equivalence clases of $M_{k}(n, x, C(f))$ is $\tilde{S}_{q}(n, k)$.

Just as in the proof of Lemma 2.1, it is sufficient to work with a fixed complete $n+1$ chain $E=\left\{E_{1} \subset E_{2} \subset \cdots \subset E_{n+1}\right\}$ of $V_{n+1}(q)$. For $f \in$ $M_{k}\left(n+1, n+1, F_{k}\right), \tilde{f}$ will denote the restriction of $f$ to $I_{n}$.

There are only two cases to consider.

Case 1. $\tilde{f} \in M_{k-1}\left(n, n+1, F_{k-1}\right)$. Since $f \in M_{k}\left(n+1, n+1, F_{k}\right)$, then $f(n+1)$ must be a line in $E_{k}-E_{k-1}$. However, any two such maps are equivalent under $\sim_{2}$. To see this, let $g$ and $h$ be any two maps obtained from 
$\tilde{f}$ by means of

$$
\begin{array}{lll}
g=\tilde{f} \text { on } I_{n}, & g(n+1)=\left\langle\left(x_{1}, x_{2}, \ldots, x_{k-1}, 1\right)\right\rangle, \\
h=\tilde{f} \text { on } I_{n}, & h(n+1)=\left\langle\left(y_{1}, y_{2}, \ldots, y_{k-1}, 1\right)\right\rangle,
\end{array}
$$

where $\left\langle\left(x_{1}, x_{2}, \ldots, x_{k-1}, 1\right)\right\rangle$ and $\left\langle\left(y_{1}, y_{2}, \ldots, y_{k-1}, 1\right)\right\rangle$ are two lines in $E_{k}-E_{k-1}$.

Let $T$ be specified by the matrix $\left(a_{i j}\right)$, where $a_{i j}$ is one on the diagonal and 0 off except that $a_{i k}=y_{i}-x_{i}$ for $i<k$.

It is not difficult to see that $T \in U_{C(f)}$ and $h=T \circ g$. That is, $h \sim_{2} g$. So in this case, $(2.3)$ yields just one $\sim_{2}$ equivalence class since we found a $T$ that did not change the image of $\tilde{f}$ while sending $g$ to $h$.

Case 2. $\tilde{f} \in M_{k}\left(n, n+1, F_{k}\right)$. Then $f(n+1)$ can be any of the $[k]$ lines in $E_{k}$. Define $g$ and $h$ as in (2.3). Then $g$ and $h$ will be equivalent if and only if we can find a $T \in U_{C(f)}$ that does not change the image of $\tilde{f}$ while sending $g$ to $h$. However, the only possible $T$ here is the identity. So we obtain [k] distinct equivalence classes in this case.

Putting both cases together and noting (1.17), we have the fact that there are $\tilde{S}_{q}(n, k) \sim_{2}$ equivalence classes of $M_{k}(n, x, C(f))$.

This completes the proof of (1.16).

We now present the proof of Proposition 1.1.

Proof. First note the following simple fact. Let $X, Y$ and $Z$ be three finite vector spaces. Suppose that $f: X \rightarrow Y$ is linear, and $T: Y \rightarrow Z$ is both linear and one-to-one. Then $f$ and $T \circ f$ have the same null space in $X$.

Let $C(f)=\left\{E_{1} \subset E_{2} \subset \cdots \subset E_{k}\right\}$. Setting $X=V_{n}(q), Y=Z=E_{k}, g$ $\in M_{k}(n, x, C(f)), T \in S_{C(f)}$, we immediately have $h=T \circ g$ implies $\Gamma h$ and $\Gamma g$ have the same null space.

Now, let us show the reverse implication. To this end, let $E=\left\{E_{1} \subset E_{2}\right.$ $\left.\subset \cdots \subset E_{k}\right\}$ and $F=\left\{F_{1} \subset F_{2} \subset \cdots \subset F_{k}\right\}$ be two different complete $k$ chains in $V_{x}(q)$.

Then, as in Definitions 2.1 and 2.2 in the proof of Lemma 2.1, there is a linear, one-to-one correspondence $\varphi$ of $E_{k}$ onto $F_{k}$ such that by setting, for $h \in M_{k}(n, x, E), \tilde{\varphi}(h)=\varphi \circ h$, we find that $\tilde{\varphi}$ is a one-to-one correspondence between $M_{k}(n, x, E)$ and $M_{k}(n, x, F)$.

Consider a map $h \in M_{k}(n, x, E)$ such that $\Gamma h$ has null space $N$, and any $T \in S_{E}$, the stabilizer group of the complete $k$ chain $E$.

By the first implication, we have that $\Gamma T \circ h$ also has null space $N$. Since $\varphi$, as defined in Definition 2.1, is linear and one-to-one, the remark at the beginning of the proof implies that $\Gamma \circ \varphi \circ T \circ h$ has null space $N$. Since $\tilde{\varphi}$ is one-to-one, we know that there are at least $(q-1)^{k} q^{\left(\frac{k}{2}\right)}$ distinct maps $g \in$ $M_{k}(n, x, E)$ with $\Gamma g$ having null space $N$. (Recall that the order of the group $S_{E}$ is $(q-1)^{k} q^{\left(\frac{k}{2}\right)}$.) 
There are $[x]_{k}$ distinct complete $k$ chains of $V_{x}(q)$, and given $g \in M_{k}(n, x)$, $C(g)$ is uniquely determined. So the collections $M_{k}(n, x, F)$ are disjoint. We have accounted for $(q-1)^{k} q^{\left(\frac{k}{2}\right)}[x]_{k}$ maps $g$ in $M_{k}(n, x)$ with $\Gamma g$ having null space $N$.

However, the null space $N$ has dimension $n-k$. Therefore, we have identified all the maps $g$ in $M_{k}(n, x)$ with $\Gamma g$ having null space $N$. That is, there are exactly $(q-1)^{k} q^{\left(\frac{k}{2}\right)}$ maps in $M_{k}(n, x, F)$ with $\Gamma g$ having null space $N$.

This completes the proof of Proposition 1.1.

3. Finite operator methods. As outlined at the end of the Introduction, as in [10], we now prove Theorems 1.1, 1.2 and 1.3.

Proof of TheOREM 1.1. We start by noting that if $n$ is any nonnegative integer, we have the identity,

$$
1=\frac{1}{e(1)} \sum_{k=0}^{\infty} \frac{1}{[k] !}=\frac{1}{e(1)} \sum_{k=n}^{\infty} \frac{1}{[k-n] !}=\frac{1}{e(1)} \sum_{k=0}^{\infty} \frac{[k]_{n}}{[k] !} .
$$

Since $L_{q}\left([x]_{n}\right)=1$, we immediately obtain

$$
L_{q}\left([x]_{n}\right)=\frac{1}{e(1)} \sum_{k=0}^{\infty} \frac{[k]_{n}}{[k] !} .
$$

Using the fact that $\left\{[x]_{k}\right\}$ form a basis for $U$, and that $L_{q}$ is a linear functional, we conclude at once that

$$
L_{q}(p(u))=\frac{1}{e(1)} \sum_{k=0}^{\infty} \frac{p(k)}{[k] !}
$$

for any polynomial $p(u)$ in the variable $q^{u}$. But this is just Theorem 1.1.

Theorem 1.2 is no more difficult to prove.

Proof. We start by observing that

$$
L_{q}\left([u][u-1]_{n}\right)=L_{q}\left([u]_{n+1}\right)=1=L_{q}\left([u]_{n}\right) .
$$

By the linearity of $L_{q}$ we obtain

$$
L_{q}([u] p(u-1))=L_{q}(p(u))
$$

where $p(u)$ is any polynomial in $q^{u}$.

Iterating (3.2), we immediately have

$$
L_{q}(p(u+k))=L_{q}\left([u]_{k} p(u)\right) .
$$

Note that $V D_{q}\left(x^{u}\right)=V\left((x q)^{u}-x^{u}\right) /(q-1) x=V[u] x^{u-1}=[u]$. Continuing in this fashion, we see that

$$
[u]_{k}=V D_{q}^{k} x^{u} .
$$

Recalling Definition 1.3, we rewrite (3.3) as 


$$
L_{q}\left(E^{k} p(u)\right)=L_{q}\left(p(u) V D_{q}^{k} x^{u}\right) .
$$

Since $L_{q}, E, D_{q}$ and $V$ are all linear, (3.5) implies that if $g$ is any polynomial in $x$, then

$$
L_{q}(g(E) p(u))=L_{q}\left(p(u) V g\left(D_{q}\right) x^{u}\right) .
$$

This finishes the proof of Theorem 1.2.

REMARK 3.1. From (3.2) and (1.21), we derive a recursive formula for $B_{q, n}$. Setting $p(u)=[u+1]^{n}$ in (3.2) gives

$$
L_{q}\left([u]^{n+1}\right)=L_{q}\left([u+1]^{n}\right)=L_{q}\left((1+q[u])^{n}\right)=\sum_{k=0}^{n}\left(\begin{array}{l}
n \\
k
\end{array}\right) q^{k} L_{q}\left([u]^{k}\right) .
$$

The identity (1.21) immediately implies that

$$
B_{q, n+1}=\sum_{k=0}^{n}\left(\begin{array}{l}
n \\
k
\end{array}\right) q^{k} B_{q, k}
$$

One formula in the generating function section is a consequence of (3.8).

The classical Charlier polynomials are essentially a consequence of (3.6) with $q=1$ and $g(x)=(x-1)^{n}$. That is, the $n$th difference operator $\Delta^{n}$ gives rise to the Charlier polynomials. As in [10], we use (3.6) with the $q n$th difference operator $\Delta_{q}^{n}$ to derive the $q$-Charlier polynomials. That is, we set

$$
\begin{aligned}
g(x) & =\left(x-q^{n-1} \cdot 1\right)\left(x-q^{n-2} \cdot 1\right) \cdots(x-q \cdot 1)(x-1) \\
& =\sum_{k=0}^{n}(-1)^{k}\left[\begin{array}{l}
n \\
k
\end{array}\right] q^{\left(\frac{k}{2}\right)} x^{n-k} .
\end{aligned}
$$

With this motivation, the proof of Theorem 1.3 is reasonably straightforward.

Proof. With $g(x)$ as above, we first see what (3.6) specializes to:

$$
g(E)=\sum_{k=0}^{n}(-1)^{k}\left[\begin{array}{l}
n \\
k
\end{array}\right] q^{\left(\frac{k}{2}\right)} E^{n-k}=\Delta_{q}^{n} .
$$

On the other hand,

$$
\begin{aligned}
V g\left(D_{q}\right) x^{u} & =\sum_{k=0}^{n}(-1)^{k}\left[\begin{array}{l}
n \\
k
\end{array}\right] q^{\left(\frac{k}{2}\right)} V D_{q}^{n-k} x^{u} \\
& =\sum_{k=0}^{n}(-1)^{k}\left[\begin{array}{l}
n \\
k
\end{array}\right] q^{\left(\frac{k}{2}\right)}[u]_{n-k} \\
& =\tilde{h}_{q, n}(u), \quad \text { by definition. }
\end{aligned}
$$

Substituting these two expressions into (3.6) gives

$$
L_{q}\left(\Delta_{q}^{n} p(u)\right)=L_{q}\left(p(u) \tilde{h}_{q, n}(u)\right)
$$


However, $L_{q}\left(p(u) \tilde{h}_{q, n}(u)\right)$ is none other than $\left\langle p(u), \tilde{h}_{q, n}(u)\right\rangle_{q}$, the $q$-inner product for the vector space of polynomials in $q^{u}$.

The left-hand side of (3.9) gives us a very efficient way to calculate this inner product. This suggests that we use $\left\{\tilde{h}_{q, n}(u)\right\}$ to be our sequence of $q$-Charlier polynomials. Let us evaluate

$$
\left\langle\tilde{h}_{q, m}(u), \tilde{h}_{q, n}(u)\right\rangle_{q} .
$$

It is sufficient to assume that $n \geqslant m$. (If not, interchange $n$ and $m$.) By (3.9) and the definition of $\tilde{h}_{q, n}(u)$, we immediately obtain

$$
\left\langle\tilde{h}_{q, m}(u), \tilde{h}_{q, n}(u)\right\rangle_{q}=\sum_{k=0}^{m}(-1)^{k}\left[\begin{array}{c}
m \\
k
\end{array}\right] q^{(k)} L_{q} \Delta_{q}^{n}[u]_{m-k}
$$

Recall that from (1.11) we know that $\Delta_{q}^{n}[u]_{k}=[k]_{n}[u]_{k-n} q^{n(u-k+n)}$. This means that

$$
\Delta_{q}^{n}[u]_{k}= \begin{cases}0 & \text { if } n>k, \\ {[n] ! q^{n u}} & \text { if } n=k .\end{cases}
$$

Putting (3.11) and (3.12) together, we immediately conclude that

$$
\left\langle\tilde{h}_{q, m}(u), \tilde{h}_{q, n}(u)\right\rangle_{q}= \begin{cases}0 & \text { if } n>k \\ L_{q}\left([n] ! q^{n u}\right) & \text { if } n=k .\end{cases}
$$

Note that $q^{u}=1+(q-1)[u]$. Therefore,

$$
\begin{aligned}
L_{q}\left([n] ! q^{n u}\right) & =[n] ! \sum_{k=0}^{n}\left(\begin{array}{l}
n \\
k
\end{array}\right)(q-1)^{k} L_{q}[u]^{k}=[n] ! \sum_{k=0}^{n}\left(\begin{array}{l}
n \\
k
\end{array}\right)(q-1)^{k} B_{q, k} \\
& \equiv f_{q}(n), \text { by definition. }
\end{aligned}
$$

Just set

$$
h_{q, n}(u)=\frac{1}{f_{q}(n)^{1 / 2}} \tilde{h}_{q, n}(u),
$$

and we have Theorem 1.3.

From (1.11) and (1.14), we derive a simple expression for $\Delta_{q}^{n}[u]^{k}$ if $n \geqslant k$. We have

$$
\begin{aligned}
\Delta_{q}^{n}[u]^{k} & =\sum_{l=0}^{k} S_{q}(k, l) \Delta_{q}^{n}[u]_{l} \\
& =\sum_{l=0}^{k} S_{q}(k, l)[l]_{n}[u]_{l-n} q^{n(u-l+n)} \\
& = \begin{cases}0 & \text { if } n>k, \\
q^{\left(\frac{n}{2}\right)}[n] ! q^{n u} & \text { if } n=k\end{cases}
\end{aligned}
$$

Note that, $\lim _{q \rightarrow 1^{+}} \Delta_{q}^{n}[u]^{n}=\Delta^{n} u^{n}=n !$. 
4. Generating function results. In this section we study two natural $q$ analogs of the exponential generating function

$$
\sum_{n=0}^{\infty} \frac{B_{n}}{n !} t^{n}=e^{\left(e^{t}-1\right)}
$$

From a combinatorial standpoint, the most natural analog of (4.1) is the Eulerian generating function,

$$
\Phi_{q}(t)=\sum_{n>0} \frac{B_{q, n}}{[n] !} t^{n}, \quad q>1 .
$$

However, recalling Remark 3.1 which gave a recursive formula for $B_{q, n}$, another natural generalization of (4.1) is

$$
\Psi_{q}(t)=\sum_{n>0} \frac{B_{q, n}}{n !} t^{n}
$$

In light of (3.8), we immediately obtain the differential-difference equation,

$$
\frac{d}{d t} \Psi_{q}(t)=e^{t} \Psi_{q}(q t) .
$$

There does not seem to be such a simple functional equation for $\Phi_{q}(t)$.

Our analysis of $\Phi_{q}(t)$ and $\Psi_{q}(t)$ depends upon Theorem 1.4, which provides an upper and lower bound for $B_{q, n}$. A direct consequence of this estimate will be a formula for the radius of convergence of $\Phi_{q}(t)$. A more detailed analysis gives information about the finite singularities of $\Phi_{q}(t)$. Except for determining its radius of convergence, we will not discuss $\Psi_{q}(t)$ any further.

Our starting point is the $q$-analog of Dobinski's equality

$$
B_{q, n}=\frac{1}{e(1)} \sum_{l>0} \frac{[l]^{n}}{[l] !} .
$$

The essential idea in the proof of Theorem 1.4 is to determine the maximal term in this series expansion of $B_{q, n}$, and for large enough $n$, bound a suitable remainder term by a certain multiple of the maximal term. As all the terms in (4.5) are nonnegative, this will bound $B_{q, n}$ between the maximal term and a multiple of the maximal term that does not increase "too fast" with $n$.

Before putting together our proof of Theorem 1.4, we need

LEMMA 4.1. If $q>1$ we have the inequality

$$
C_{q}=\exp \frac{-(2 q-1)}{2(q-1)^{2}}<\prod_{n=1}^{\infty}\left(1-1 / q^{n}\right) .
$$

Proof. Note that $\left|-1 / q^{n}\right|<1 / q<1$, so that $\log \left(1-1 / q^{n}\right)<0$ and is defined. We may then write $\Pi_{k=1}^{n}\left(1-1 / q^{k}\right)$ as $\exp \sum_{k=1}^{n} \log \left(1-1 / q^{k}\right)$. 
We now estimate $\log \left(1-1 / q^{n}\right)$ from below.

$$
\begin{aligned}
\log \left(1-1 / q^{n}\right) & =\left(-1 / q^{n}\right)-\left(-1 / q^{n}\right)^{2} / 2+\left(-1 / q^{n}\right)^{3} / 3-\cdots \\
& \geqslant\left(-1 / q^{n}\right)\left(1-\left(-1 / q^{n}\right) / 2+\left(-1 / q^{n}\right)^{2} / 2-\cdots\right) \\
& =\left(-1 / q^{n}\right)\left(1+\frac{\left(-1 / q^{n}\right)}{2}\left(\frac{1}{1-1 / q^{n}}\right)\right) \\
& >-1 / q^{n}\left(1+\frac{1}{2(q-1)}\right) .
\end{aligned}
$$

As a result of this lower bound, we have

$$
\sum_{k=1}^{n} \log \left(1-1 / q^{k}\right) \geqslant-\sum_{k=1}^{n} 1 / q^{k}\left(1+\frac{1}{2(q-1)}\right)=\frac{-(2 q-1)}{2(q-1)^{2}} .
$$

Consequently,

$$
\prod_{k=1}^{n}\left(1-1 / q^{k}\right) \geqslant \exp \frac{-(2 q-1)}{2(q-1)^{2}}
$$

and the result follows.

We are now in a position to prove Theorem 1.4.

Proof. We first find the dominant term in the series for $B_{q, n}$ given in (4.5). For convenience, set

$$
a_{l}=\frac{1}{e(1)} \frac{[l]^{n}}{[l] !}
$$

It is immediate that the ratio of successive terms is

$$
\frac{a_{l+1}}{a_{l}}=q^{n-l-1}(q-1)\left(1-\frac{1}{q^{l+1}}\right)^{n-1} /\left(1-\frac{1}{q^{l}}\right)^{n} .
$$

As $l$ increases, $q^{n-l-1}(q-1)$ decreases. So, for $n$ sufficiently large, there is a unique value of $l$, say $l_{0}$, such that $q^{n-l_{0}-1}(q-1)>1$ and $q^{n-l_{0}-2}(q-1)$ $<1$. It turns out that $l_{0}=n-k_{q}-1$, where $k_{q}$ is the largest integer contained in $-\log (q-1) / \log q$.

It is not difficult to see that for a fixed $n$ and $l$ increasing, (1$\left.1 / q^{l+1}\right)^{n-1} /\left(1-1 / q^{l}\right)^{n}$ is strictly decreasing.

We observe next that

$$
\lim _{n \rightarrow \infty}\left(1-\frac{1}{q^{n-k_{q}}}\right)^{n-1} /\left(1-\frac{1}{q^{n-k_{q}-1}}\right)^{n}=1 .
$$

Putting both of these facts together, we may deduce that for any $l>0$, 


$$
1 \leqslant\left(1-\frac{1}{q^{l+1}}\right)^{n-1} /\left(1-\frac{1}{q^{l}}\right)^{n},
$$

and given any $\varepsilon>0$, for $n$ large enough and $l>n-k_{q}$, we have

$$
1 \leqslant\left(1-\frac{1}{q^{l+1}}\right)^{n-1} /\left(1-\frac{1}{q^{l}}\right)^{n} \leqslant 1+\varepsilon .
$$

From (4.8) we conclude that $0 \leqslant l \leqslant n-k_{q}-1, a_{l+1}>a_{l}$.

We claim that $a_{l+1}<a_{l}$ when $n$ is sufficiently large and $l>n-k_{q}$. This would imply that the dominant term in (4.5) is $a_{n-k_{q}}$.

Indeed, since $q^{k_{q}-1}(q-1)<1$, and $q^{n-l-1}(q-1)$ is decreasing in $l$, (4.9) implies that

$$
q^{n-l-1}(q-1)\left(1-\frac{1}{q^{l+1}}\right)^{n-1} /\left(1-\frac{1}{q^{l}}\right)^{n}<1,
$$

when $n$ is large and $l>n-k_{q}$. That is, $a_{l+1}<a_{l}$ for $l>n-k_{q}$.

We now estimate a remainder term in (4.5) by a certain multiple of the maximal term.

It is not hard to see that

$$
\begin{aligned}
\frac{[l]^{n}}{[l] !} & =\frac{1}{(q-1)^{n}} \frac{(q-1)^{l} q^{\left((2 n-1) l-l^{2}\right) / 2}\left(1-\frac{1}{q^{l}}\right)^{n}}{(1-1 / q)\left(1-1 / q^{2}\right) \cdots\left(1-1 / q^{l}\right)} \\
& \leqslant \frac{1}{(q-1)^{n}}(q-1)^{l} q^{\left((2 n-1) l-l^{2}\right) / 2} \cdot \frac{1}{C_{q}}
\end{aligned}
$$

by Lemma 4.1. But this is just

$$
\frac{1}{(q-1)^{n}}(q-1)^{l} q\left(\left[(2 n+1) l-l^{2}\right]-2 l\right) / 2 \frac{1}{C_{q}},
$$

which is less than

$$
\frac{1}{(q-1)^{n}}\left(\frac{q-1}{q}\right)^{l} \frac{1}{C_{q}},
$$

provided $l>2 n+2$. Setting $l=(2 n+2)+m$, we obtain

$$
\frac{[l]^{n}}{[l] !}<\frac{1}{C_{q}} \frac{(q-1)^{n+2}}{q^{2 n+2}}\left(\frac{q-1}{q}\right)^{m} .
$$

But this gives us

$$
\sum_{l=2 n+2}^{\infty} \frac{[l]^{n}}{[l] !}<\frac{(q-1)^{n+2}}{q^{2 n+1}} \frac{1}{C_{q}}
$$


Note that $\left[n-k_{q}\right]^{n} /\left[n-k_{q}\right] ! \geqslant 1$ and $\lim _{n \rightarrow \infty}\left[(q-1)^{n+2} / q^{2 n+1}\right]=0$. So for $n$ sufficiently large,

$$
\frac{1}{C_{q}} \frac{(q-1)^{n+2}}{q^{2 n+1}}<1 \leqslant \frac{\left[n-k_{q}\right]^{n}}{\left[n-k_{q}\right] !} .
$$

Therefore, by (4.5), and the fact that $\left[n-k_{q}\right]^{n} /\left[n-k_{q}\right]$ ! is the dominant term, $e(1) B_{q, n} \leqslant(2 n+2)\left[n-k_{q}\right]^{n} /\left[n-k_{q}\right]$ ! for all large $n$. In any event, $a_{l}>0$ for all $l$. Therefore, $\left[n-k_{q}\right]^{n} /\left[n-k_{q}\right] !<e(1) B_{q, n}$. Putting these last two estimates together completes the proof of Theorem 1.4.

As for the convergence of $\Phi_{q}(t)$, we have

THEOREM 4.1. Suppose that.

$$
\Phi_{q}(t)=\sum_{n>0} \frac{B_{q, n}}{[n] !} t^{n}, \quad q>1 .
$$

Then the radius of convergence of $\Phi_{q}(t)$ is $q / q-1$. Furthermore, if $|z|=$ $q / q-1$,

$$
\liminf _{n \rightarrow \infty}\left|\frac{B_{q, n}}{[n] !} z^{n}\right|>\frac{1}{e(1)} .
$$

That is, $\Phi_{q}(t)$ diverges on its circle of convergence.

Proof. The main idea of the proof is to show that the $\lim _{\inf }{ }_{n \rightarrow \infty}$ and lim sup $\operatorname{su}_{n \rightarrow \infty}$ of $\left(B_{q, n} /[n] !\right)^{1 / n}$ converge to the same thing, by applying the estimates for $B_{q, n}$ provided by Theorem 1.4.

Note that if $c>0$ is a constant, then $\lim _{n \rightarrow \infty} c^{1 / n}=1$. Also, recall that $\lim _{n \rightarrow \infty}(n+1)^{1 / n}=1$.

Consequently, by Theorem 1.4, we will be done if we can show that the $\lim \inf _{n \rightarrow \infty}$ and $\lim \sup _{n \rightarrow \infty}$ of $\left(\left[n-k_{q}\right]^{n} /\left[n-k_{q}\right] ![n] !\right)^{1 / n}$ both converge to $(q-1) / q$.

After some algebraic simplication, it is not difficult to see that

$$
\left[n-k_{q}\right]^{n} /\left[n-k_{q}\right] ![n] !
$$

is equal to

$$
\frac{\left(q^{n-k_{q}}-1\right)^{n}(q-1)^{n}}{q^{n(n+1)-n k_{q}}(q-1)^{k_{q}} q^{\left(k_{q}^{2}-k_{q}\right) / 2} \prod_{k=1}^{n}\left(1-1 / q^{k}\right) \prod_{k=1}^{n-k_{q}}\left(1-1 / q^{k}\right)} .
$$

By Lemma 4.1, 


$$
\prod_{k=1}^{n}\left(1-\frac{1}{q^{k}}\right)_{k=1}^{n-k_{q}}\left(1-\frac{1}{q^{k}}\right)
$$

is larger than $\left(c_{q}\right)^{2}$.

However, as $q<1$,

$$
\prod_{k=1}^{n}\left(1-\frac{1}{q^{k}}\right)_{k=1}^{n-k_{q}}\left(1-\frac{1}{q^{k}}\right)<1 .
$$

Keeping in mind that $c_{q}$ and $k_{q}$ are constants independent of $n$, we will be finished if we show that

$$
\lim _{n \rightarrow \infty}\left[\frac{\left(q^{n-k_{q}}-1\right)^{n}(q-1)^{n}}{q^{n(n+1)-n k_{q}}}\right]^{1 / n}=(q-1) / q
$$

Indeed, this is simply,

$$
\lim _{n \rightarrow \infty} \frac{\left(q^{n-k_{q}}-1\right)(q-1)}{q^{\left(n+1-k_{q}\right)}}=(q-1) \lim _{n \rightarrow \infty}\left(\frac{1}{q}-\frac{1}{q^{n+1-k_{q}}}\right),
$$

which is $(q-1) / q$.

This completes the radius of convergence calculation.

Now it is easy to show that $\Phi_{q}(t)$ diverges everywhere on its circle of convergence.

If $|z|=q /(q-1)$, then

$$
\begin{aligned}
\frac{B_{q, n}}{[n] !} \frac{q^{n}}{(q-1)^{n}} & >\frac{[n]^{n}}{([n] !)^{2}} \frac{q^{n}}{(q-1)^{n}} \frac{1}{e(1)} \\
& =\frac{1}{e(1)} \frac{\left(q^{n}-1\right)^{n} \cdot q^{n}}{q^{n(n+1)}\left(\prod_{k=1}^{n}\left(1-\frac{1}{q^{k}}\right)\right)^{2}} \\
& >\frac{1}{e(1)}\left(1-\frac{1}{q^{n}}\right)^{n}
\end{aligned}
$$

Note that $\lim _{n \rightarrow \infty}\left(1-1 / q^{n}\right)^{n}=1$. Therefore, if $|z|=q /(q-1)$, then $\lim \inf _{n \rightarrow \infty}\left|B_{q, n} z^{n} /[n] !\right|>1 / e(1)$.

This completes the proof of Theorem 4.1.

It is not hard to see that for $|t|<q /(q-1), \Phi_{q}(t)$ can be written in integral form as,

$$
\Phi_{q}(t)=\int_{-\infty}^{+\infty} e(t[x]) d P_{q}(x)
$$

Letting $q \rightarrow 1^{+}$, we obtain the well-known result, 


$$
e^{\left(e^{t}-1\right)}=\int_{-\infty}^{+\infty} e^{t x} d P(x),
$$

where $P(x)$ is a Poisson distribution with $\lambda=1$. It is reasonable to regard (4.11) as a $q$-analog of a characteristic function.

We now deduce the radius of convergence of

$$
\Psi_{q}(t)=\sum_{n>0} \frac{B_{q, n}}{n !} t^{n}
$$

for $q>0$.

Recall that $\Psi_{1}(t)=e^{\left(e^{t}-1\right)}$ and that $B_{q, n+1}=\sum_{k=0}^{n}\left(\begin{array}{l}n \\ k\end{array}\right) q^{k} B_{q, k}$.

The comparison test immediately implies that the radius of convergence of $\Psi_{q}(t)$ is infinite when $0<q<1$.

By the same methods as in Theorem 4.1, it is not difficult to treat the case $q>1$.

Just as in (4.10), $\left[n-k_{q}\right]^{n} /\left[n-k_{q}\right] ! n !$ is equal to

$$
\left[\frac{1}{n !} \frac{\left(q^{n-k_{q}}-1\right)^{n}}{q^{\left(n^{2}+n-2 n k_{q}\right) / 2}}\right] \cdot \frac{1}{(q-1)^{k_{q}} \prod_{k=1}^{n-k_{q}}\left(1-1 / q^{k}\right) q^{\left(k_{q}^{2}-k_{q}\right) / 2}} .
$$

Just as in Theorem 4.1, it is sufficient to look at

$$
\lim _{n \rightarrow \infty}\left[\frac{1}{n !} \frac{\left(q^{n-k_{q}}-1\right)^{n}}{q^{\left(n^{2}+n-2 n k_{q}\right) / 2}}\right]^{1 / n}
$$

By Stirling's approximation to $n$ ! this is simply

$$
\lim _{n \rightarrow \infty}\left(\frac{\left(\frac{n}{e}\right)^{n} \sqrt{2 \pi n}}{n !}\right)^{1 / n} \lim _{n \rightarrow \infty} \frac{\left(q^{n-k_{q}}-1\right)}{q^{-k_{q}} q^{(n+1) / 2}\left(\frac{n}{e}\right) \sqrt{(2 \pi n)^{1 / n}}}
$$

The first of these limits is 1 and the second is

$$
e \lim _{n \rightarrow \infty} \frac{q^{n}\left(1-1 / q^{n-k_{q}}\right)}{q^{(n+1) / 2} \cdot n} \rightarrow \infty \quad \text { as } n \rightarrow \infty .
$$

Therefore, $\Psi_{q}(t)$ has 0 radius of convergence for $q>1$.

We close this section by discussing the finite singularities of $\Phi_{q}(t)$.

Since $B_{q, n}>0$ and the radius of convergence of $\Phi_{q}(t)$ is finite, it follows by a theorem in complex analysis that $z=q /(q-1)$ is a singularity of $\Phi_{q}(t)$.

It turns out that $z=q /(q-1)$ is a simple pole of $\Phi_{q}(t)$. Indeed, we prove that there are no more singularities on the circle of convergence by showing that the function $(1-(q-1) t / q) \Phi_{q}(t)$ has a radius of convergence equal to 
$q^{2} /(q-1)$. This is not obvious as there is no simple functional equation for $\Phi_{q}(t)$. One must put together a proof similar to Theorem 4.1. In this case, the algebra is much more complicated. However, once we have the analog of Dobinski's equality set up, the analysis follows essentially the same pattern as before. We will be content with deriving the Dobinski equalities that we need as well as one new estimate that reduces the analysis in this case to that in the proof of Theorem 4.1.

We first note that

$$
\left(1-\frac{(q-1)}{q} t\right) \Phi_{q}(t)=1+\sum_{n>1}\left(\frac{B_{q, n}}{[n] !}-\frac{(q-1)}{q} \frac{B_{q, n-1}}{[n-1] !}\right) t^{n} .
$$

Define $A_{n}$ by means of the equation

$$
A_{n}=\left(\frac{B_{q, n}}{[n] !}-\frac{(q-1)}{q} \frac{B_{q, n-1}}{[n-1] !}\right) .
$$

By (4.5), it is not hard to see that $A_{n+1}$ equals

$$
\frac{1}{e(1)} \frac{1}{[n+1] !}\left\{\sum_{l>0} \frac{1}{[l] ! q}\left(q[l+1]^{n}-(q-1)[n+1] \cdot[l]^{n}\right)\right\} \text {. }
$$

After some algebraic simplifications, we find that

$$
q[l+1]^{n}-(q-1)[n+1][l]^{n}
$$

is equal to

$$
\frac{q^{1+n l}}{(1-1 / q)^{n}}\left\{\left(\left(1-\frac{1}{q^{l+1}}\right)^{n}-\left(1-\frac{1}{q^{l}}\right)^{n}\right)+\frac{1}{q^{n+1}}\left(1-\frac{1}{q^{l}}\right)^{n}\right\} .
$$

It is clear that this expression is positive. Therefore $A_{n}$ may be written as a sum of positive numbers.

Incidently, as a result of $A_{n}$ being nonnegative, we immediately obtain

$$
\frac{B_{q, n}}{[n] B_{q, n-1}} \geqslant \frac{q-1}{q} .
$$

In view of the fact that $\Phi_{q}(t)$ has radius of convergence $q / q-1$, it seems reasonable to conjecture that the sequence $\left\{B_{q, n} /[n] B_{q, n-1}\right\}$ is monotone decreasing to $(q-1) / q$ as $n$ increases. That is, that the $q$-Bell numbers, as well as the classical ones, are almost log-concave. This is borne out by numerical evidence but we have not yet put together a proof.

Putting (4.13), (4.14), (4.15) and (4.16) together, we find that

$$
A_{n+1}=\frac{1}{e(1)} \frac{1}{[n+1] !}\left(S_{n+1,1}+S_{n+1,2}\right)
$$


where

$$
S_{n+1,1}=\sum_{l>0} \frac{q^{n l}}{[l] !(1-1 / q)^{n}}\left(\left(1-\frac{1}{q^{l+1}}\right)^{n}-\left(1-\frac{1}{q^{l}}\right)^{n}\right),
$$

and

$$
S_{n+1,2}=\sum_{l>0} \frac{q^{n l}}{[l] !(1-1 / q)^{n}}\left(\frac{1}{q^{n+1}}\left(1-\frac{1}{q^{l}}\right)^{n}\right) .
$$

The main idea is to apply the analytical techniques of Theorem 4.1 to each of the sums $S_{n+1,1}$ and $S_{n+1,2}$, separately. That is, find the maximal term in the sum and estimate a suitable remainder in terms of this maximum. We then obtain estimates for the $\lim \inf _{n \rightarrow \infty}$ and $\lim \sup _{n \rightarrow \infty}$ of the $n$th root of the sum. These two quantifiers turn out to be equal and our radius of convergence result follows.

This construction may be applied directly to the sum $S_{n+1,2}$. However, to apply these methods to $S_{n+1,1}$, we need to know that

$$
\begin{aligned}
\frac{(q-1)}{q(l+1)} n\left(1-\frac{1}{q^{l}}\right)^{n-1} & <\left(1-\frac{1}{q^{l+1}}\right)^{n}-\left(1-\frac{1}{q^{l}}\right)^{n} \\
& <\frac{(q-1)}{q(l+1)} n\left(1-\frac{1}{q^{l+1}}\right)^{n-1} .
\end{aligned}
$$

This is an immediate consequence of the mean value theorem applied to the function $f(x)=x^{n}$ over the integral $\left(1-1 / q^{l}\right)<x<\left(1-1 / q^{l+1}\right)$.

Not only is $q^{2} /(q-1)$ the radius of convergence of $(1-(q-1) t / q) \Phi_{q}(t)$, but since $A_{n} \geqslant 0$, it is also a singularity.

From combinatorial considerations, it is reasonable to conjecture that $z=q^{2} /(q-1)$ is a simple pole.

Moreover, we conjecture that the only finite singularities of $\Phi_{q}(t)$ are of the form

$$
q^{m} /(q-1), \quad m>1 .
$$

To make this conjecture more plausible, we study two other generating functions that exhibit the above type of behavior for their finite singularities, while counting classes of objects related to those enumerated by $\Phi_{q}(t)$.

Recall that

$$
\begin{aligned}
([x])^{n} & =\sum_{k=0}^{n} S_{q}(n, k)[x]_{k}, \quad \text { and } \\
B_{q, n} & =\sum_{k=0}^{n} S_{q}(n, k) .
\end{aligned}
$$


$B_{q, n}$ is the number of mappings of $I_{n}$ into the "lines" of $V_{n}(q)$, that are associated with a fixed $n$-complete chain $E$ of $V_{n}(q)$. That is, $B_{q, n}$ counts the number of maps in $\operatorname{RG}(n, q, E)$.

The "affine" analog of (4.20) is given by

$$
\begin{aligned}
\left(q^{x}\right)^{n} & =\sum_{k=0}^{n}\left[\begin{array}{l}
n \\
k
\end{array}\right](q-1)^{k} q^{\left(\frac{k}{2}\right)}[x]_{k}, \quad \text { and } \\
F_{q, n} & =\sum_{k=0}^{n}\left[\begin{array}{l}
n \\
k
\end{array}\right](q-1)^{k} q^{\left(\frac{k}{2}\right)} .
\end{aligned}
$$

Here $F_{q, n}$ is the number of mappings of $I_{n}$ into the "points" of $V_{n}(q)$ that are associated with a fixed $n$-complete chain of $V_{n}(q)$. That is, the number of "affine" restricted growth functions.

The analog of $\Phi_{q}(t)$ is the Eulerian generating function

$$
F_{q}(t)=\sum_{n>0} \frac{F_{q, n}}{[n] !} t^{n} .
$$

In [6], Jay Goldman and G. C. Rota discussed the generating function

$$
G_{q}(t)=\sum_{n>0} \frac{\left(\sum_{k=0}^{n}\left[\begin{array}{l}
n \\
k
\end{array}\right]\right)}{[n] !} t^{n} .
$$

Note that $\sum_{k=0}^{n}\left[\begin{array}{l}n \\ k\end{array}\right]$ is the total number of subspaces of $V_{n}(q)$.

Since $\Phi_{q}(t), F_{q}(t)$ and $G_{q}(t)$ are generating functions which count related classes of objects, it is reasonable to expect that their finite singularities be of a similar type.

It turns out that there are simple closed expressions for both $F_{q}(t)$ and $G_{q}(t)$. From these expressions, it is not difficult to determine all the finite singularities.

These closed expressions immediately follow from two theorems about $q$-Appell polynomials that appear in [2]. For completeness, we repeat them here.

THEOREM 4.2. A polynomial set $\left\{P_{n}(x)\right\}$ is $q$-Appell if and only if there is a set of constants $\left\{a_{k}\right\}$ such that $a_{0} \neq 0$,

$$
P_{n}(x)=\sum_{k=0}^{n}\left[\begin{array}{l}
n \\
k
\end{array}\right] a_{n-k} x^{k}
$$

THEOREM 4.3. A polynomial set $\left\{P_{n}(x)\right\}$ is q-Appell if and only if there is a formal power series

$$
A(t)=\sum_{k=0}^{\infty} \frac{a_{k}}{[k] !} t^{k}, \quad a_{0} \neq 0
$$

such that 


$$
A(t) e(x t)=\sum_{n=0}^{\infty} P_{n}(x) t^{n} /[n] !
$$

Setting $x=1$ in Theorems 4.2 and 4.3, as well as noting that $\left[\begin{array}{c}n \\ k\end{array}\right]=\left[\begin{array}{c}n \\ n-k\end{array}\right]$, it is not difficult to show that

$$
F_{q}(t)=\left(\sum_{n>0} \frac{q^{(n)}(q-1)^{n}}{[n] !} t^{n}\right) \cdot\left(\sum_{n>0} \frac{t^{n}}{[n] !}\right),
$$

and

$$
G_{q}(t)=\left(\sum_{n>0} \frac{t^{n}}{[n] !}\right)^{2} .
$$

By the definitions of $e(x)$ and $E(x)$ given in (1.23) and (1.24), these two equalities become

$$
\begin{aligned}
F_{q}(t) & =E((q-1) t) e(t), \\
G_{q}(t) & =(e(t))^{2} .
\end{aligned}
$$

For $q>1$, we have the identities

$$
\begin{gathered}
E((q-1) t)=\prod_{i>1}\left(1-\frac{(q-1)^{2}}{q^{i}} t\right)^{-1}, \\
e(t)=\prod_{i>1}\left(1-\frac{(q-1)}{q^{i}} t\right) .
\end{gathered}
$$

From (4.25), (4.27), and (4.28), it follows that the only finite singularities of $F_{q}(t)$ are simple poles at the points

$$
q^{i} /(q-1)^{2}, \quad i>1 .
$$

It turns out that for $|q|<1, e(t)=\Pi_{i>0}\left(1-q^{i}(q-1) t\right)^{-1}$. This implies that $G_{q}(t)=\Pi_{i>0}\left(1-q^{i}(q-1) t\right)^{-2}$. This formula was derived in [6]. Therefore, the only finite singularities of $G_{q}(t)$ for $|q|<1$, are double poles at the points

$$
1 / q^{i}(q-1), \quad i>0 .
$$

Though these examples make our conjecture plausible, a proof seems to require a new fundamental property of Eulerian generating functions.

Though we did not discuss it in this paper, there is a remarkable class of rank and order preserving mappings of the lattice of subspaces of $V_{n}(q)$, onto the lattice of subsets of $I_{n}$ which gives a set-theoretical reason for why the projective form of $q$-identities approaches binomial identities when we let $q \rightarrow 1$. These matters and their consequences will be developed in a future publication. 
REMARK ADDED IN PROOF. Hahn [7] has considered $q$-analogs of the classical orthogonal polynomials but has not given enough details so that it is easy to see what the $q$-Charlier polynomials should be. Recently, they have been given explicitly by Andrews and Askey [1].

\section{REFERENCES}

1. G. E. Andrews and R. A. Askey, The classical and discrete orthogonal polynomials and their q-analogs (to appear).

2. W. A. Al-Salam, q-Appell polynomials, Ann. Mat. Pura Appl. (4) 77 (1967), 31-45.

3. L. Carlitz, q-Bernoulli numbers and polynomials, Duke Math. J. 15 (1948), 987-1000.

4. _ On abelian fields, Trans. Amer. Math. Soc. 35 (1933), 122-136.

5. G. Dobinski, Summirungder Reihe $\Sigma n^{m} / n$ ! für $m=1,2,3,4,5, \ldots$, Grunert's Archiv 61 (1877), 333-336.

6. J. Goldman and G. C. Rota, The number of subspaces of a vector-space, Recent Progress in Combinatorics, 1969, pp. 75-83.

7. W. Hahn, Über Orthogonalpolynome, die q-Differenzen-gleichungen genügen, Math. Nachr. 2 (1949), 4-34.

8. G. Hutchinson, Partitioning algorithms for finite sets, Comm. ACM 6 (1963), 613-614.

9. S. Milne, Restricted growth functions and incidence relations of the lattice of partitions of an n-set, Advances in Math. 26 (1977), 290-305.

10. G. C. Rota, The number of partitions of a set, Amer. Math. Monthly 71 (1964), 498-504.

11. S. G. Williamson, Ranking algorithms for lists of partitions, SIAM J. Comput. 5 (1976), 602-617.

Department of Mathematics, Yale University, New Haven, Connecticut 06520

Current address: Department of Mathematics, Texas A \& M University, College Station, Texas 77843 